\title{
Experimental and Theoretical Investigations of PAN Molecular Weight Increase in Precipitation Polymerization as a Function of $\mathrm{H}_{2} \mathrm{O} / \mathrm{DMSO}$ Ratio
}

\author{
Jing Zhang ${ }^{1}$, Fengjing Bu ${ }^{1}$, Yongqiang Dai ${ }^{1}$, Liwei Xue ${ }^{1}$, Zhixian $\mathrm{Xu}^{2}$, Seung Kon Ryu ${ }^{3}$ and Riguang Jin ${ }^{1, \wedge}$ \\ ${ }^{1,}$ State Key Laboratory of Chemical Resource Engineering, Beijing University of Chemical Technology, Beijing, 100029, China \\ ${ }^{2}$ Dept. of Chemistry and Chemical Engineering, Anyang Normal University, Henan, 455000, China \\ ${ }^{3}$ Dept. of Chemical Engineering, Chungnam National University, Daejeon 305-764, Korea \\ ^e-mail: jin.riguang@163.com, skryu@cnu.ac.kr \\ (Received February 8, 2010; Accepted March 10, 2010)
}

\begin{abstract}
The precipitation polymerization of acrylonitrile (AN) was carried out in a mixture solution of dimethyl sulfoxide (DMSO) and water at $50 \sim 65^{\circ} \mathrm{C}$ using $\alpha, \alpha$-azobisisobutyronitrile (AIBN) as an initiator. The increased molecular weight polyacrylonitrile (PAN) was prepared with increasing the $\mathrm{H}_{2} \mathrm{O} / \mathrm{DMSO}$ ratio from 10/90 to 80/20. The viscosity average molecular weight of $\mathrm{H}_{2} \mathrm{O}$ /DMSO solvent was 4.4 times larger than that of $\mathrm{H}_{2} \mathrm{O} / \mathrm{DMF}$ solvent, and precipitation polymerization was accelerlated due to the far decreased chain transfer effect of DMSO. Based on the experimental results, the increased PAN molecular weight was regarded as the summation of two mechanisms: i) particle-particle aggregation and ii) particle-radical attachment. The theoretical equation derived from the mechanisms was well coincided with the experimental results showing the linear relationship between the viscosity average molecular weight and the $\mathrm{H}_{2} \mathrm{O} / \mathrm{DMSO}$ ratio.
\end{abstract}

Keywords : PAN, Precipitation polymerization, Molecular weight increase, $\mathrm{H}_{2} \mathrm{O} / \mathrm{DMSO}$ ratio

\section{Introduction}

As a type of high-performance inorganic fiber, carbon fiber has lots of good superiorities such as specific strength, high modulus, lightfastness, corrosion resistance, fatigue, creep resistance, electrical conductivity, excellent heat transfer performance, and etc. [1,2]. When temperature is as high as $2000^{\circ} \mathrm{C}$, carbon fiber is the only material that the strength does not fall in an inert environment. It is well known that the properties of high performance polyacrylonitrile (PAN) based carbon fiber are determined strongly by the nature of precursor, and preparing high molecular weight polymer is one of the effective ways to obtain high strength fiber [3-6]. In general, a second monomer such as acrylic ester and a third monomer such as itaconic acid were added to monomer in order to increase the molecular weight of PAN in solution polymerization.

Recently, precipitation polymerization is increasing the interests in the preparation of high molecular weight PAN due to the advantage of small chain transfer reaction than solution polymerization in using a second monomer [7-10]. Bahrami [11] reported the effect of $\mathrm{H}_{2} \mathrm{O}$ /dimethyl formamide (DMF) on the conversion rate and intrinsic viscosity during precipitation polymerization using acrylonitrile and acrylic acid as monomers and $\mathrm{H}_{2} \mathrm{O}$ and DMF as mixed solvent system. Wang [12] studied the effect of monomer concentration, initiator concentration, ratio of $\mathrm{H}_{2} \mathrm{O}$ /dimethyl sulfoxide (DMSO) on the precipitation polymerization, and suggested that precipitation polymerization was more effective than solution polymerization in preparation of high performance carbon fibers. Zhang [13] also studied the effect of precipitation polymerization conditions on the conversion rate using $\mathrm{H}_{2} \mathrm{O} / \mathrm{DMSO}$ mixture solution. Wang [14] reported the relationship between apparent activation energy and the ratio of $\mathrm{H}_{2} \mathrm{O} / \mathrm{DMSO}$. However, there are few studies on the relationship between molecular weight increase of PAN and $\mathrm{H}_{2} \mathrm{O} / \mathrm{DMSO}$ ratio, especially relating to the theoretical mechanism of PAN molecular weight increase.

Therefore, the purpose of this study is experimental and theoretical investigations of PAN molecular weight increase in precipitation polymerization as functions of $\mathrm{H}_{2} \mathrm{O} / \mathrm{DMSO}$ ratio and intrinsic viscosity. To obtain the purpose, i) precipitation polymerization of acrylonitrile was carried out by changing the solvent ratio of $\mathrm{H}_{2} \mathrm{O} / \mathrm{DMSO}$ at different temperature, ii) molecular weight and intrinsic viscosity of the obtained polymer were measured in DMF solution, and iii) model mechanism of precipitation polymerization was suggested in relation between molecular weight increase of PAN and $\mathrm{H}_{2} \mathrm{O} / \mathrm{DMSO}$ ratio. 


\section{Experimental}

\subsection{Materials}

Acrylonitrile (AN, Beijing Chemical Reagents Co.) was freed from inhibitors by distillation at $76 \sim 78^{\circ} \mathrm{C}$ before polymerization. $\alpha, \alpha^{\prime}$-azobisisobutyronitrile (AIBN) was purified by recrystallization. Dimethyl sulfoxide (DMSO) and deionized water $\left(\mathrm{H}_{2} \mathrm{O}\right)$ were used as polymerization solvents. Dimethyl formamide (DMF) solution was used to compare the intrinsic viscosity of the polymer with that of DMSO solution.

\subsection{Precipitation polymerization}

Precipitation polymerization of AN was carried out in a $1.0 \mathrm{~L}$ three-necked glass reactor by adding $0.3 \mathrm{~L}$ different $\mathrm{H}_{2} \mathrm{O} / \mathrm{DMSO}$ ratio from $10 / 90$ to $80 / 20$ (wt/wt) as solvent to $0.1 \mathrm{~L} \mathrm{AN}$ for $2 \mathrm{~h}$ at $50,55,60$ and $65^{\circ} \mathrm{C}$ under pure nitrogen atmosphere. After polymerization, enough distilled water was added into the reactor to precipitate the produced polymer. The precipitated polymer was washed with distilled water and ethanol successively, filtered with Buchner funnel, and dried under vacuum at $40^{\circ} \mathrm{C}$ till a constant weight was obtained.

\subsection{Intrinsic viscosity measurement}

Intrinsic viscosity [ $\eta$ ] of the obtained polymers was measured in DMF solution using an Ubbelohde viscometer in a water bath at constant temperature of $35 \pm 0.1^{\circ} \mathrm{C}$ following the Kashyap's method [15]. The viscosity average molecular weight $M_{\eta}$ was calculated from the following equation [16]:

$$
[\eta]=2.78 \times 10^{-4} \mathrm{M}_{\eta}^{0.76}
$$

\section{Results and Discussion}

\subsection{Experimental study}

Fig. 1 shows the SEM images of PAN obtained from $\mathrm{H}_{2} \mathrm{O}$ / DMSO ratio of $10 / 90$ and $80 / 20$ in precipitation polymerization at $55^{\circ} \mathrm{C}$ for $2 \mathrm{~h}$. The morphology of PAN obtained from small ratio of $\mathrm{H}_{2} \mathrm{O}$ (10/90) shows loosely aggregated large particles. While that of obtained from $80 / 20$ shows big spheres composed of tightly aggregated small particles. These images suggested that there might be immense number of micro-droplet monomers in $80 / 20$ ratio solvent which was divided by $\mathrm{H}_{2} \mathrm{O}$ at the beginning of precipitation polymerization. And these immense micro-droplet monomers proceeded the precipitation polymerization, then generated plenty of precipitation particles, finally resulted in the

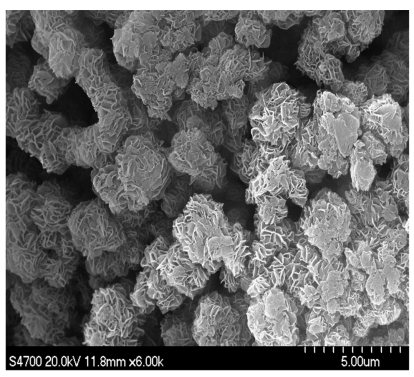

(a)

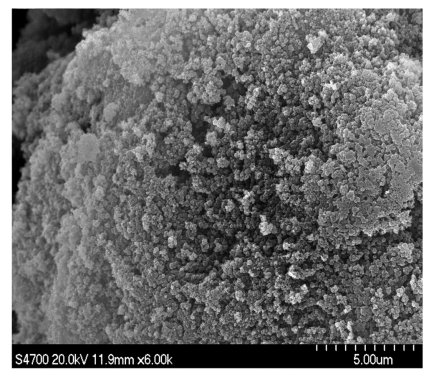

(b)
Fig. 1. SEM images of PAN obtained from $\mathrm{H}_{2} \mathrm{O} / \mathrm{DMSO}$ ratio of (a) $10 / 90$ and (b) $80 / 20$ in precipitation polymerization at $55^{\circ} \mathrm{C}$ for $2 \mathrm{~h}$.

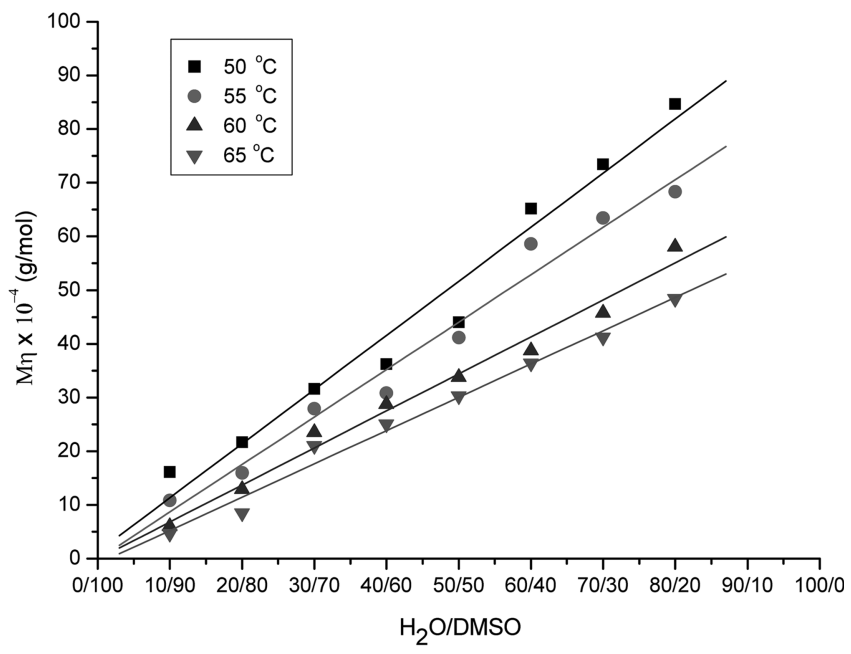

Fig. 2. Relationship between $\mathrm{H}_{2} \mathrm{O} / \mathrm{DMSO}$ ratio and viscosity average molecular weights of PAN.

increase of intrinsic viscosity and viscosity average molecular weight. The size and shapes of aggregation were depended on the $\mathrm{H}_{2} \mathrm{O} / \mathrm{DMSO}$ ratio, and higher molecular weight PAN precursor can be obtained from increasing the $\mathrm{H}_{2} \mathrm{O} / \mathrm{DMSO}$ ratio.

Fig. 2 shows the viscosity average molecular weights of PAN as a function of $\mathrm{H}_{2} \mathrm{O} / \mathrm{DMSO}$ ratio obtained at 50, 55, 60 and $65^{\circ} \mathrm{C}$. The viscosity molecular weight linearly increased as the increase of $\mathrm{H}_{2} \mathrm{O} / \mathrm{DMSO}$ ratio from 10/90 to $80 / 20$. This was because the number of micro-droplet monomer increased as the increase of $\mathrm{H}_{2} \mathrm{O} / \mathrm{DMSO}$ ratio, and the increased micro-droplet were aggregated until they became big precipitation particles, resulted in the molecular weight increase, which made the solution more turbid and viscous. Wang [12] and Zhang [13] have reported the similar results on the effect of $\mathrm{H}_{2} \mathrm{O} / \mathrm{DMSO}$ ratio. On the other hand, the viscosity average molecular weight decreased as the increase of reaction temperature at the fixed ratio of $\mathrm{H}_{2} \mathrm{O} / \mathrm{DMSO}$. This was believed high 


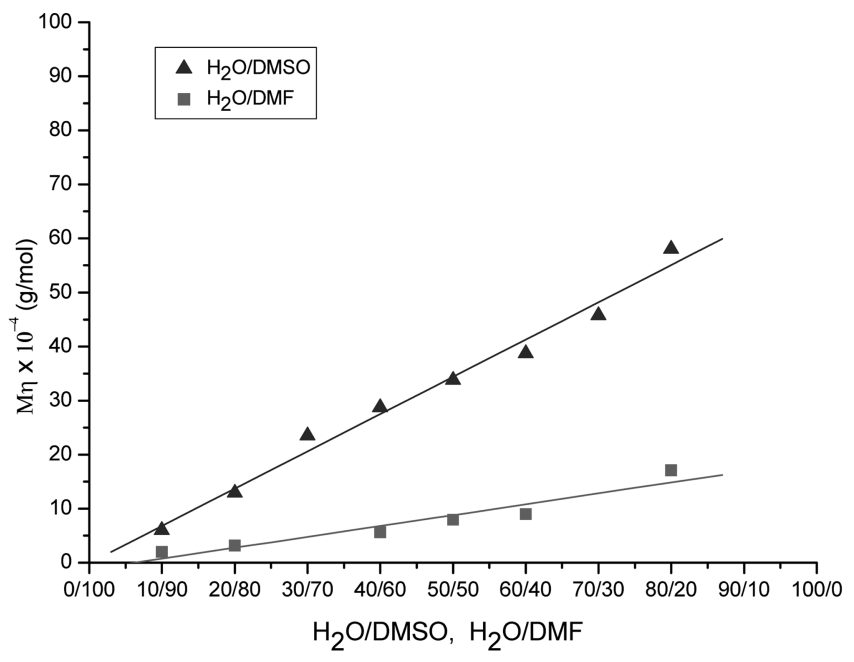

Fig. 3. Viscosity average molecular weights of $\mathrm{PAN}$ from $\mathrm{H}_{2} \mathrm{O} /$ DMSO and $\mathrm{H}_{2} \mathrm{O} / \mathrm{DMF}$ solvent in precipitation polymerization at $60^{\circ} \mathrm{C}$.

reaction temperature promoted the suspension of microdroplet rather than particles aggregation, resulted in the decrease of viscosity.

Fig. 3 shows the viscosity average molecular weights of the PAN as a function of $\mathrm{H}_{2} \mathrm{O} / \mathrm{DMSO}$ and $\mathrm{H}_{2} \mathrm{O} / \mathrm{DMF}$ ratio obtained from precipitation polymerization at $60^{\circ} \mathrm{C}$. The viscosity average molecular weight of $\mathrm{H}_{2} \mathrm{O} / \mathrm{DMSO}$ solvent was 4.4 times larger than that of $\mathrm{H}_{2} \mathrm{O} / \mathrm{DMF}$ solvent. Bahrami [11] reported very similar data to those of our $\mathrm{H}_{2} \mathrm{O} / \mathrm{DMF}$ shown in Fig. 3. The higher value of $\mathrm{H}_{2} \mathrm{O} / \mathrm{DMSO}$ system was due to the decreased chain transfer effect of the DMSO. The chain transfer constant of water is almost zero and that of DMSO is much less than DMF [10]. Therefore, $\mathrm{H}_{2} \mathrm{O}$ / DMSO solvent system is far better than $\mathrm{H}_{2} \mathrm{O} / \mathrm{DMF}$ system for the precipitation polymerization to obtain increased molecular weight of PAN.

\subsection{Theoretical study}

Based on the experimental results, summation of two mechanisms, particle-particle aggregation and particleradical attachment can be suggested in the formation of the increased molecular weight PAN.

Mechanism I was regarded as a particle-particle aggregation. In the precipitation polymerization of acrylonitrile, the obtained polymer was not dissolved in the $\mathrm{H}_{2} \mathrm{O} / \mathrm{DMSO}$ mixture solution. Once it was generated, it became nascent particle and suspended in the solution. As the core, the nascent particle aggregated with other particles until it became a larger particle in the precipitation polymerization. This process can be regarded as n-dimensional aggregation as shown in Fig. 4.

The number of micro-droplet monomer increased as the increase of $\mathrm{H}_{2} \mathrm{O} / \mathrm{DMSO}$ ratio due to the division of droplet by $\mathrm{H}_{2} \mathrm{O}$, which made the solution more turbid and viscous. From this consideration, the following equation can be proposed.

$$
[P] \propto\left[\mathrm{H}_{2} \mathrm{O} / \mathrm{DMSO}\right]
$$

Where, $[\mathrm{P}]$ is the concentration of the nascent precipitation particle, and following hypothesis can be suggested. $F(\mathrm{P})$ is the number of aggregated monomers in multi-dimensional direction in precipitation polymerization. When the nascent particle aggregated with other one particle, $F(\mathrm{P})$ will be proportional to $[\mathrm{P}]$ showing the following equation.

$$
F(\mathrm{P})_{1}=k_{11}[\mathrm{P}]^{1}
$$

Where, $F(\mathrm{P})$ is the number of aggregated monomers in multi-dimensional direction in precipitation polymerization,

$$
\begin{aligned}
& k_{i j} \text { : aggregation rate constant, } \\
& \mathrm{i}: \text { dimension, } \\
& \mathrm{j} \text { : serial number of particles. }
\end{aligned}
$$

When the nascent precipitation particle aggregated with other two particles, $F(\mathrm{P})$ will be proportional to the square of

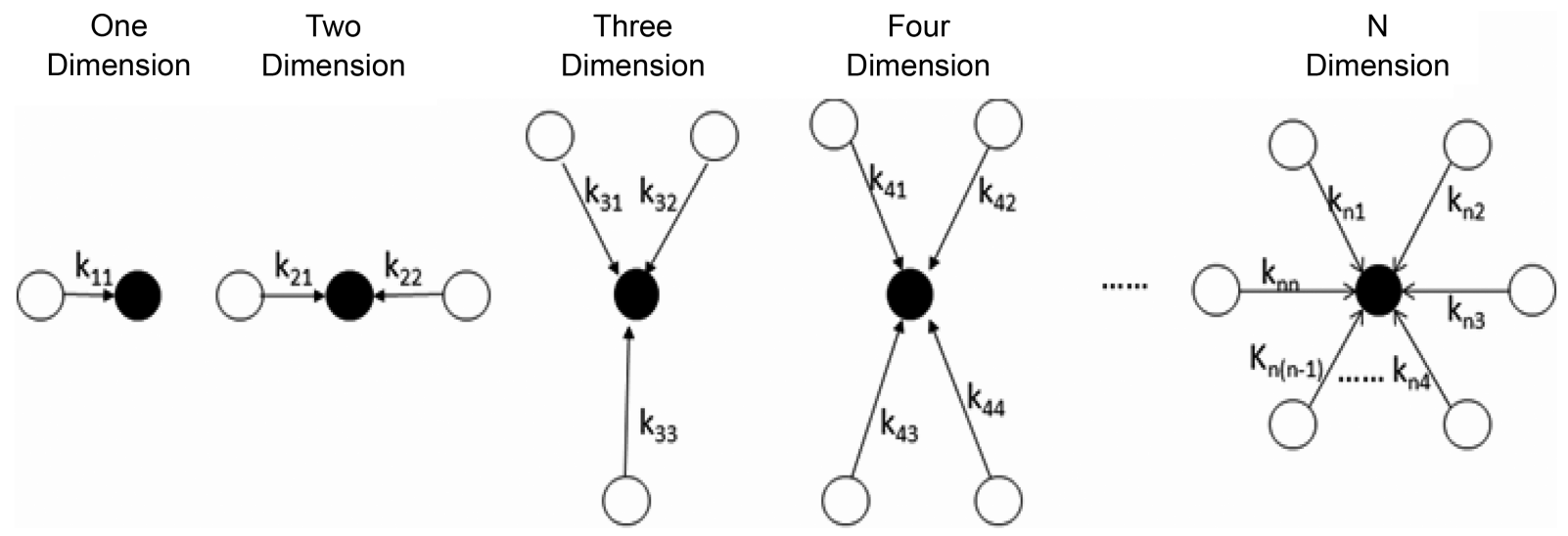

Fig. 4. Aggregation of nascent PAN particles in precipitation polymerization. 
$[\mathrm{P}]$, and the aggregation rate constant of the second particle will be half of the first particle in the contribution of aggregation. Therefore, the $F(\mathrm{P})_{2}$ can be expressed as the following equation.

$$
F(\mathrm{P})_{2}=\frac{k_{21} \times k_{21}}{2 \times 1}[\mathrm{P}]^{2}=\frac{k_{22} \times k_{22}}{2 !}[\mathrm{P}]^{2}
$$

When the nascent precipitation particle aggregated with other three particles, $F(\mathrm{P})$ will be proportional to the cube of $[\mathrm{P}]$, and the aggregation rate constant of the third particle will be one-third of the first particle in contribution of aggregation.

$$
F(\mathrm{P})_{3}=\frac{k_{31} \times k_{31} \times k_{31}}{3 \times 2 \times 1}[\mathrm{P}]^{3}=\frac{k_{31} \times k_{31} \times k_{31}}{3 !}[\mathrm{P}]^{3}
$$

When the nascent precipitation particle aggregated with other $\mathrm{n}$ particles, $F(\mathrm{P})$ will be proportional to $[\mathrm{P}]^{\mathrm{n}}$, and the aggregation rate constant of the $\mathrm{n}^{\text {th }}$ particle will be one- $\mathrm{n}^{\text {th }}$ of the first particle in contribution of aggregation.

$$
\begin{aligned}
& F(\mathrm{P})_{n}=\frac{k_{n n} \times k_{n(n-1)} \times \cdots k_{n 2} \times k_{n 1}}{n \times(n-1) \times \cdots 2 \times 1}[\mathrm{P}]^{n} \\
& =\frac{k_{n n} \times k_{n(n-1)} \times \cdots k_{n 2} \times k_{n 1}}{n !}[\mathrm{P}]^{n}
\end{aligned}
$$

If there was no particle to aggregate, $F(\mathrm{P})$ will be proportional to $[\mathrm{P}]^{0}$, and expressed as the following equation.

$$
F(\mathrm{P})_{0}=k_{00}[\mathrm{p}]^{0}=k_{00}
$$

Therefore, all the aggregated nascent particles will be the summation of $F(\mathrm{P})_{1}+F(\mathrm{P})_{2}$----- $+F(\mathrm{P})_{\mathrm{n}}$, and the relationship between $\mathrm{F}(\mathrm{P})$ and $[\mathrm{P}]$ can be obtained as follows.

$$
\begin{aligned}
& F(P)=\sum_{i=1}^{n} F(\mathrm{P})_{i}=k_{00}+k_{11}[\mathrm{P}]+\frac{k_{21} \times k_{22}}{2 !}[\mathrm{P}]^{2} \\
& \frac{k_{31} \times k_{32} \times k_{33}}{3 !}[\mathrm{P}]^{3}+\cdots+\frac{k_{n n} \times k_{n(n-1)} \times \cdots \times k_{n 2} \times k_{n 1}}{n !}[\mathrm{P}]^{n}
\end{aligned}
$$

If each nascent precipitation particle has same ability for precipitation and $\mathrm{k}_{\mathrm{ij}}$ is the function of temperature, it can be regarded to $\mathrm{k}_{\mathrm{ij}}=\mathrm{k}_{\mathrm{ji}}=\mathrm{k}_{00}=\mathrm{k}_{11}=\cdots \mathrm{k}_{\mathrm{nn}}$, and the equation (8) can be simplified as follows.

$$
F(\mathrm{P})=\sum_{i=1}^{n} F(\mathrm{P})_{i}=k+k[\mathrm{P}]+\frac{k^{2}}{2 !}[\mathrm{P}]+\cdots+\frac{k^{n}}{n !}[\mathrm{P}]^{n}
$$

The equation is exactly part of the Taylor series expansion, so the equation (9) can be changed as the following form:

$$
F(\mathrm{P})=e^{K_{1}[P]}
$$

Therefore, the total aggregated number of nascent precipitation particles is

$$
F(\mathrm{P})=e^{K_{1}[P]} \propto Q
$$

Where $\mathrm{Q}$ is the quantity of precipitates and $K_{1}$ is the

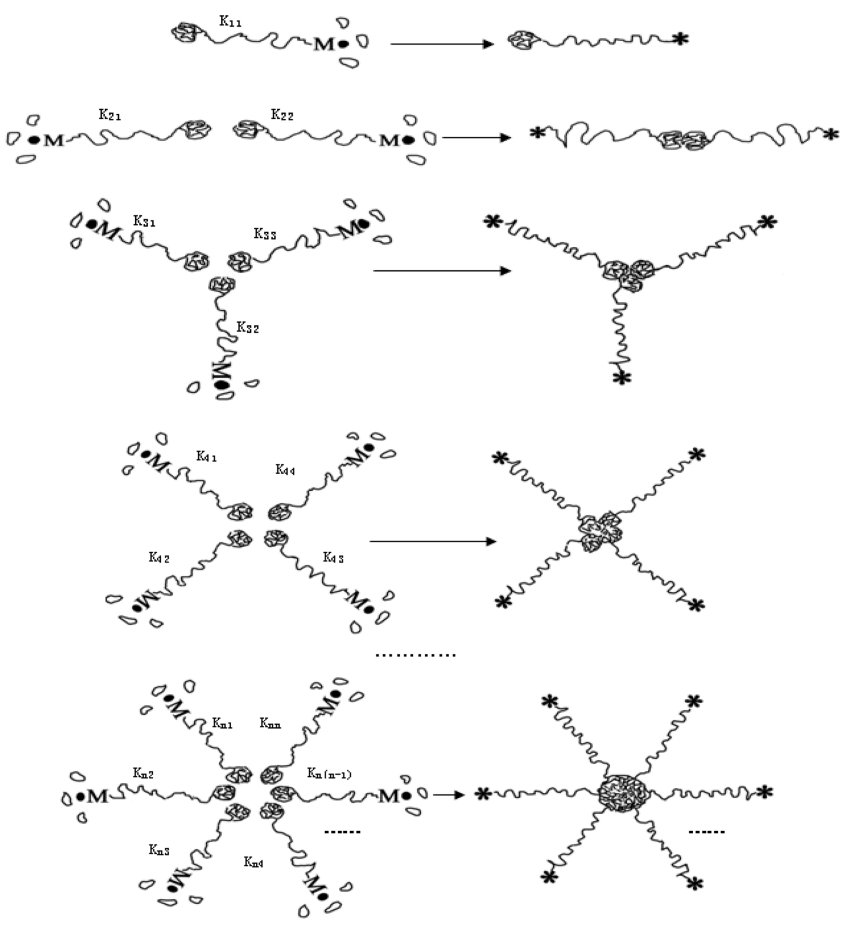

Fig. 5. Attachment of particles to PAN free radicals in precipitation polymerization.

overall aggregation rate constant.

Mechanism II was regarded as particle-radical attachment. When the weight ratio of $\mathrm{H}_{2} \mathrm{O} / \mathrm{DMSO}$ was small, the number of nascent precipitation particle will be limited. The propagation radical $(M \bullet)$ can mainly move in a large range in DMSO solvent, resulted in the reaction easily terminate and viscosity average molecular weight $\left(\mathrm{M}_{\eta}\right)$ small. When the ratio of $\mathrm{H}_{2} \mathrm{O} / \mathrm{DMSO}$ increased, the number of nascent precipitation particles also rapidly increased. In this process, each propagation radical will be sited on everywhere of nascent precipitation particles. These propagation radicals only react in DMSO, and the propagation radicals may not easily move with the decrease of DMSO. Therefore, the termination opportunity of adjacent two propagation radicals will be reduced. In this case, propagation radical will have much more opportunity to react and form lager molecule. Finally, molecular weight will be increased constantly as the increase of $\mathrm{H}_{2} \mathrm{O} / \mathrm{DMSO}$ ratio as shown in Fig. 5 .

Based on the physical concept above, it can be put forward a model similar to the previous. When the nascent particle attached to one other particle, $F\left(M_{\bullet}\right)$ will be proportional to $[\mathrm{M} \bullet$ and expressed as the following equation.

$$
F(\mathrm{M} \cdot)_{1}=k_{11}[\mathrm{M} \cdot]^{1}
$$

Where, $F(\mathrm{M} \bullet)$ : number of attachment monomer in multidimensional direction in precipitation polymerization, 
$k_{11}:$ attachment rate constant,

i : dimension,

$\mathrm{j}$ : serials number of particles.

When the nascent particle reacted with two other particles, $F\left(M_{\bullet}\right)$ will be proportional to the square of $[\mathrm{M} \bullet]$, and the attachment rate constant of the second particle will be half of the first particle in the contribution of attachment.

$$
F(\mathrm{M} \cdot)_{2}=\frac{k_{21} \times k_{22}}{2 \times 1}[\mathrm{M} \cdot]^{2}=\frac{k_{21} \times k_{22}}{2 !}[\mathrm{M} \cdot]^{2}
$$

When the nascent particle attached to three other particles, $F(M \bullet)$ will be proportional to the cube of [M॰], and the attachment rate constant of the third particle will be onethird of the first particle in contribution of attachment.

$$
F(\mathrm{M} \cdot)=\frac{k_{31} \times k_{32} \times k_{33}}{3 \times 2 \times 1}[\mathrm{M} \cdot]^{3}=\frac{k_{31} \times k_{32} \times k_{33}}{3 !}[\mathrm{M} \cdot]^{3}
$$

When the nascent monomer particle attached to $\mathrm{n}$ other particles, $F\left(M_{\bullet}\right)$ will be proportional to $\left[\mathrm{M}_{\bullet}\right]^{\mathrm{n}}$, and the attachment rate constant of the $n^{\text {th }}$ particle will be one- $n^{\text {th }}$ of the first particle in contribution of attachment.

$$
\begin{aligned}
& F(\mathrm{M} \cdot)_{n}=\frac{k_{n n} \times k_{n(n-1)} \times \cdots \times k_{n 2} \times k_{n 1}}{n \times(n-1) \times \cdots \times 2 \times 1}[\mathrm{M} \cdot]^{n} \\
& =\frac{k_{n n} \times k_{n(n-1)} \times \ldots \times k_{n 2} \times k_{n 1}}{n !}[\mathrm{M} \cdot]^{n}
\end{aligned}
$$

If there is no monomer particle for attachment, $F\left(\mathrm{M}_{\bullet}\right)$ will be proportional to $[\mathrm{M} \bullet]^{0}$.

$$
F(M \cdot)_{0}=k_{00}[M \cdot]^{0}=k_{00}
$$

Therefore, all the attached nascent monomer particles will be the summation of $F(M \bullet)_{1}+F(M \bullet)_{2}+\ldots+F(M \bullet)_{\mathrm{n}}$ and the relationship between $F\left(\mathrm{M}_{\bullet}\right)$ and [M•] can be obtained as follows.

$$
\begin{aligned}
& F(\mathrm{M} \cdot)=\sum_{i=1}^{n} F(\mathrm{M} \cdot)_{i}=k_{00}+k_{11}[\mathrm{M} \cdot]+\frac{k_{21} \times k_{22}}{2 !}[\mathrm{M} \cdot]^{2}+ \\
& \frac{k_{31} \times k_{32} \times k_{33}}{3 !}[\mathrm{M} \cdot]^{3}+\cdots+\frac{k_{n n} \times k_{n(n-1)} \times k_{n 2} \times k_{n 1}}{n !}[\mathrm{M} \cdot]^{n}
\end{aligned}
$$

If each first born particle has same ability for precipitation and $k_{i j}$ is the function of temperature, it can be regarded as $k_{i j}=k_{j i}=k_{00}=k_{11}=\cdots k_{n n}$, and the equation (17) can be simplified as follow as :

$$
\begin{aligned}
& F(\mathrm{M} \cdot)=\sum_{i=1}^{n} F(\mathrm{M} \cdot)_{i}=k+k[\mathrm{M} \cdot]+\frac{k^{2}}{2 !}[\mathrm{M} \cdot]^{2} \\
& +\ldots+\frac{k^{n}}{n !}[\mathrm{M} \cdot]^{n}
\end{aligned}
$$

The equation (18) was exactly part of the Taylor series expansion, so it can be changed as the following form:

$$
F(M \cdot)=e^{k_{2}[\mathrm{M} \cdot]}
$$

The total attached monomer of nascent precipitation particles are

$$
F(\mathrm{M} \cdot)=e^{K_{2}[\mathrm{M} \cdot]} \propto Q
$$

Where $\mathrm{Q}$ is the quantity of precipitates and $K_{1}$ is the overall attachment rate constant.

Therefore, from (11) and (20):

$$
e^{K_{1}[P]} \propto e^{K_{2}[\mathrm{M} \cdot]}
$$

Taking logarithm on both sides of the equation:

$$
[\mathrm{P}] \propto[\mathrm{M} \cdot] \propto \mathrm{M \eta}
$$

The viscosity average molecular weight can be calculated by the summation of (2) and (22), and the final equation can be suggested as follows.

$$
[\mathrm{M} \eta]=\mathrm{K}_{3}+\mathrm{K}_{4}\left[\mathrm{H}_{2} \mathrm{O} / \mathrm{DMSO}\right]
$$

Where $\mathrm{K}_{3}$ is inversely proportional to $\mathrm{K}_{2}$ and $K_{4}=\frac{K_{1}}{K_{2}}$

From (23), it can be found that there is a linear relationship between the viscosity average molecular weight and the $\mathrm{H}_{2} \mathrm{O} / \mathrm{DMSO}$ ratio. Therefore, the theoretical equation derived from the mechanism was well coincided with the experimental results showing the linear relationship between the viscosity average molecular weight and the $\mathrm{H}_{2} \mathrm{O} / \mathrm{DMSO}$ ratio.

\section{Conclusions}

In the precipitation polymerization PAN molecular weight increase was depended on the increase of $\mathrm{H}_{2} \mathrm{O} / \mathrm{DMSO}$ ratio due to the increased micro-droplets, which made the solution more turbid and viscous, resulted in the molecular weight increase. Using $\mathrm{H}_{2} \mathrm{O} / \mathrm{DMSO}$ solvent system was far better than using $\mathrm{H}_{2} \mathrm{O} / \mathrm{DMF}$ system in precipitation polymerization and obtained increased molecular weight PAN precursor because of decreased chain transfer effect of DMSO. Based on the experimental results, the increased molecular weight of PAN was regarded as the summation of two mechanisms: particle-particle aggregation and particleradical attachment. The theoretical equation derived from the mechanisms can be used to estimate the viscosity average molecular weight of the newly polymerized polyacrylonitrile (PAN) precursor.

\section{References}

[1] Wu, S. Z. Polym. Adv. Technol. 2005, 16, 642.

[2] Bajaj, P. Indian J. of Appl. Polym. Sci. 2002, 79, 1640.

[3] Ho, C. T.; Chung, Carbon 1990, 28, 831. 
[4] Mittal, J.; R. B. Mathur, R. B.; Bahl, O. P. Carbon 1997, 35, 1713.

[5] Hou, C. J. of Appl. Polym. Sci. 2006, 101, 2095.

[6] Hou, C.; Liu, J.; Yang, L. J. of Appl. Polym. Sci. 2006, 100, 4679.

[7] Chen, J. C.; Harrison, I. R. Carbon 2002, 40, 25.

[8] Wilkinson, K. American Patant, 96P 39552, 1996212212, USA.

[9] Jia, D. M.; Yang, M. Y. Chemical Fiber of Beijing 1998, 2 , 1.

[10] Cai, H. S. Acta Polymerica Sinica 1999, 5, 1.
[11] Bahrami, S. H. Iranian Polym. Jourmal 2005, 14, 1032.

[12] Wang, Y. Z.; Zhu, B. Journal of Shangdong University 2002, 33, 362.

[13] Zhang, W. X. "PAN-based carbon fiber", Donghua University Press, 2005, 12.

[14] Wang, C. G European Polym. Journal 2006, 42, 1093.

[15] Kashyap, A. K.; Kalpagam, V. J. Polym Sci Part: Ploym Chem Edit 1979, 17, 1243.

[16] Roychen, J.; Surekha, D.; Animesh, K. R. Polym Int. 1991, 26, 89. 